The authors have identified a previously unrecognized integrin $\alpha 6 \beta 4$-expressing AEC subset that proliferates and differentiates into multiple respiratory epithelial cell types in vitro and in vivo. The field can look forward to future studies identifying the roles of these $\alpha 6 \beta 4$-positive AECs in the pathogenesis of human lung disease. Elucidation of the mechanisms controlling their proliferation, differentiation, and ability to produce functional lung tissue will be highly relevant to the pathogenesis of acute and chronic lung disease. It will also provide a framework from which to develop new strategies to enhance lung regeneration for the treatment of life-threatening common pulmonary disorders.

\section{Acknowledgments}

The authors acknowledge funding from the NIH (grant HL090156 to J.A. Whitsett; grant HL084151 to V.V. Kalinichenko).
Address correspondence to: Jeffrey A. Whitsett, Cincinnati Children's Hospital Medical Center, Division of Pulmonary Biology, MLC 7029, 3333 Burnet Avenue, Cincinnati, Ohio 45229-3039, USA. Phone: 513.803.2790; Fax: 513.636.7868; E-mail: jeff.whitsett@cchmc.org.

1. Hogan BL, Yingling JM. Epithelial/mesenchymal interactions and branching morphogenesis of the lung. Curr Opin Genet Dev. 1998;8(4):481-486.

2. Morrisey EE, Hogan BL. Preparing for the first breath: genetic and cellular mechanisms in lung development. Dev Cell. 2010;18(1):8-23.

3. Cardoso WV. Molecular regulation of lung development. Annu Rev Physiol. 2001;63:471-494.

4. Whitsett JA, Wert SE, Weaver TE. Alveolar surfactant homeostasis and the pathogenesis of pulmonary disease. Annu Rev Med. 2010;61:105-119.

5. Chapman HA, et al. Integrin $\alpha 6 \beta 4$ identifies an adult distal lung epithelial population with regenerative potential in mice. J Clin Invest. 2011; 121(7):2855-2862.

6. Warburton D, et al. Lung organogenesis. Curr Top Dev Biol. 2010;90:73-158.

7. Whitsett JA, Wert SE, Trapnell BC. Genetic disorders influencing lung formation and function at birth.
Hum Mol Genet. 2004;13(spec no 2):R207-R215.

8. Perl AK, Wert SE, Nagy A, Lobe CG, Whitsett JA. Early restriction of peripheral and proximal cell lineages during formation of the lung. Proc Natl Acad SciU S A. 2002;99(16):10482-10487.

9. Adamson IY, Bowden DH. The type 2 cell as progenitor of alveolar epithelial regeneration. A cytodynamic study in mice after exposure to oxygen. Lab Invest. 1974;30(1):35-42.

10. Rawlins EL, et al. The role of Scgb1a1+ Clara cells in the long-term maintenance and repair of lung airway, but not alveolar, epithelium. Cell Stem Cell. 2009;4(6):525-534.

11. Rock JR, et al. Basal cells as stem cells of the mouse trachea and human airway epithelium. Proc Natl Acad Sci U S A. 2009;106(31):12771-12775

12. Kim CF, et al. Identification of bronchioalveolar stem cells in normal lung and lung cancer. Cell. 2005;121(6):823-835

13. McQualter JL, Yuen K, Williams B, Bertoncello I. Evidence of an epithelial stem/progenitor cell hierarchy in the adult mouse lung. Proc Natl Acad Sci US A. 2010;107(4):1414-1419.

14. Green MD, et al. Generation of anterior foregut endoderm from human embryonic and induced pluripotent stem cells. Nat Biotechnol. 2011;29(3):267-272.

15. Kajstura J, et al. Evidence for human lung stem cells. NEngl J Med. 2011;364(19):1795-1806.

\title{
PON-dering differences in HDL function in coronary artery disease
}

\author{
Chieko Mineo and Philip W. Shaul
}

Division of Pulmonary and Vascular Biology, Department of Pediatrics, University of Texas Southwestern Medical Center, Dallas, Texas, USA.

\begin{abstract}
HDL cholesterol activates endothelial cell production of the atheroprotective signaling molecule NO, and it promotes endothelial repair. In this issue of the JCI, Besler et al. provide new data indicating that HDL from stable coronary artery disease (CAD) or acute coronary syndrome patients inhibits rather than stimulates endothelial NO synthesis and endothelial repair. This may be related to decreased HDL-associated paraoxonase 1 (PON1) activity. These observations support the concept that the cardiovascular impact of HDL is not simply related to its abundance, and the translation of the present findings to prospective studies of CAD risk and to evaluations of HDLtargeted therapeutics is a logical future goal.
\end{abstract}

\section{HDL and cardiovascular disease}

Numerous epidemiological studies indicate that the risk of cardiovascular disease is inversely related to HDL cholesterol levels (1). In addition, mice with elevated LDL due to the expression of a human apolipoprotein $\mathrm{B}$ transgene have more severe atherosclerosis if they also have low HDL due to deficiency of the major apolipoprotein of HDL, apoA-I (2).

Conflict of interest: The authors have declared that no conflict of interest exists.

Citation for this article: J Clin Invest. doi:10.1172/ JCI57671.
Furthermore, providing apoA-I or HDL improves vascular disease in hypercholesterolemic animal models $(3,4)$, and studies in humans have suggested that the infusion of apoA-I mimetics or reconstituted HDL particles has the potential to reverse atherosclerosis (5). However, genetically based variations in HDL levels in humans do not correspond directly with relative incidence or severity of cardiovascular disease (6), and therapies that effectively cause increases in HDL levels have not yielded clear-cut decreases in cardiovascular disease $(7,8)$. This was particularly the case in a trial of torcetrapib, an inhibitor of cholesteryl ester transfer protein, which yielded a $72 \%$ increase in HDL levels but an actual increase in cardiovascular events (9). Thus, the cardiovascular impact of HDL is not simply related to the abundance of the lipoprotein, and there is a great need for increased understanding of the biological actions of HDL and the development of assays to assess HDL function in humans. In the current issue of the JCI, Besler and colleagues provide important new insights into the atheroprotective actions of HDL in a comparison of the endothelial functions of HDL obtained from healthy individuals and HDL from patients with stable coronary artery disease (CAD) or acute coronary syndrome (10), designated as $\mathrm{HDL}_{\mathrm{Healthy}}$ and $\mathrm{HDL}_{\mathrm{CAD}}$, respectively, throughout this commentary.

\section{Biological actions of HDL}

The classical function of HDL is to mediate cholesterol efflux from peripheral tissues and cells such as macrophages and transport it back to the liver in a process known as reverse cholesterol transport (RCT). 

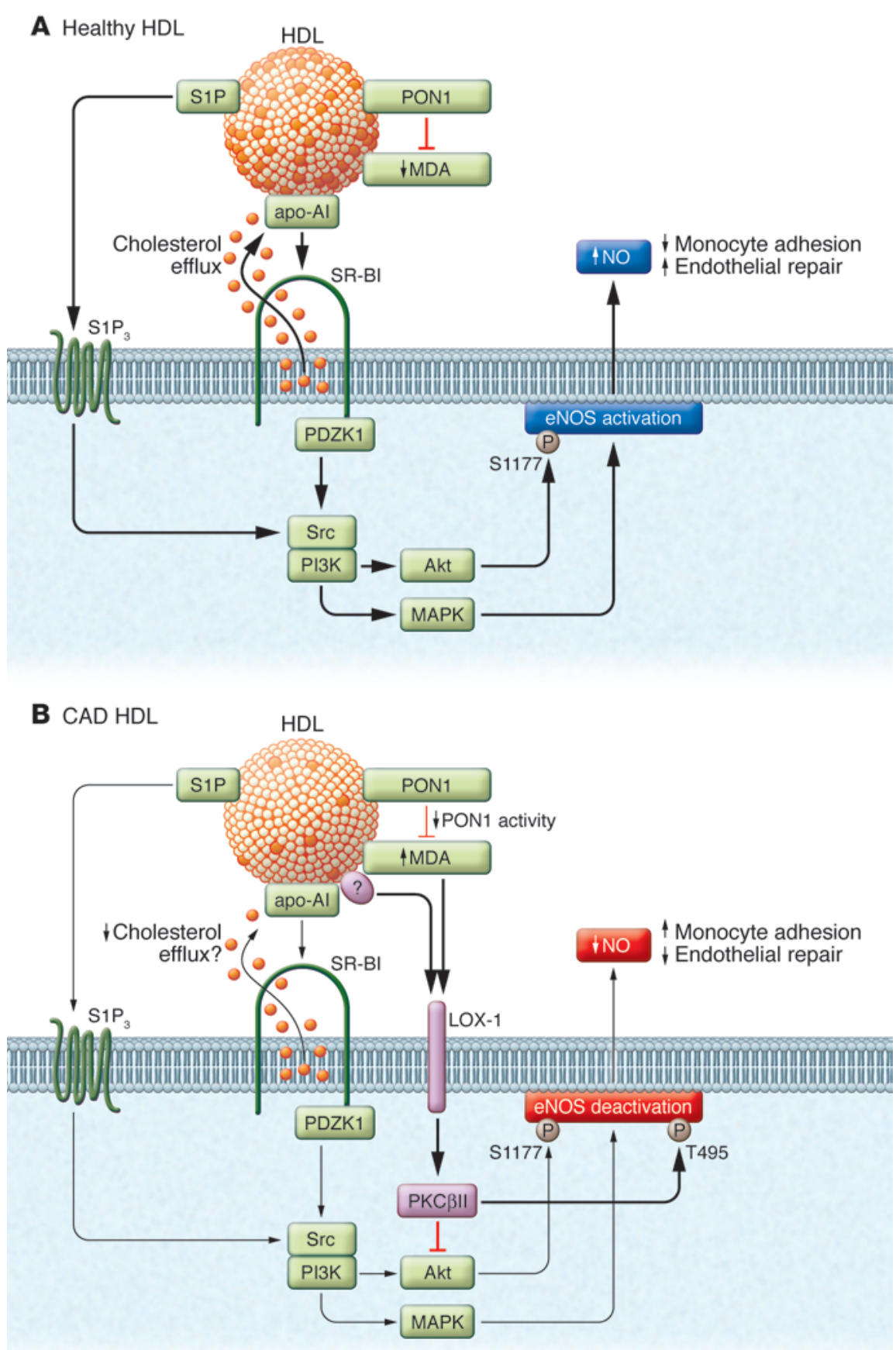

RCT likely plays an important role in the cardiovascular protective capacity of HDL (11). A measure of the "cholesterol efflux capacity" of HDL was recently reported to be inversely associated with atherosclerosis in humans even after adjusting for HDL cholesterol levels (12).

In addition to promoting RCT, it is now well appreciated that HDL has direct actions on the endothelium that may also contribute to the cardiovascular protective characteristics of the lipoprotein (13). In endothelial cells, HDL from healthy individuals activates the production of the antiatherosclerotic and antithrombotic signaling molecule NO by eNOS (14), it blunts adhesion molecule expression (13), attenuates tissue factor and E-selectin expression (13), and promotes endothelial cell migration, thereby optimizing endothelial repair and the integrity of the intimal layer (15). eNOS activation by HDL entails apoA-Idependent binding of the lipoprotein to scavenger receptor class B, type I (SR-BI)

\section{Figure 1}

Changes in endothelial HDL action in coronary artery disease. (A) HDL Healthy stimulates eNOS through multiple mechanisms. HDL binding to SR-BI via apoA-I causes cholesterol efflux (orange circles) that is sensed by SR$\mathrm{BI}$, leading to PDZK1-dependent activation of Src family kinase(s), PI3K, and Akt, which phosphorylates eNOS Ser1177 and thereby increases enzyme activity. SR-BI-, Src-, and PI3K-dependent Erk MAPK activation is also required for eNOS activation by HDL. HDLassociated $\mathrm{S} 1 \mathrm{P}$ and related molecules may activate the lysophospholipid receptor $\mathrm{S}_{1} \mathrm{P}_{3}$ to stimulate eNOS. HDL Healthy contains active PON1, which suppresses the formation of oxidized lipids and lipoproteins such as MDA. The net effect of eNOS activation by HDL Healthy is to blunt endothelial cell-monocyte adhesion and promote endothelial repair. (B) HDL $\mathrm{HAD}_{\mathrm{C}}$ activates inhibitory signaling that suppresses eNOS activation. HDL CAD $_{\text {Das }}$ reduced PON1 activity that potentially leads to greater formation of MDA, which activates LOX-1 and thereby stimulates $\mathrm{PKC} \beta$. PKC $\beta$ inhibits stimulatory Akt and eNOS phosphorylation events, and it enhances the inhibitory phosphorylation of eNOS at Thr495. As a result, $\mathrm{HDL}_{\text {CAD }}$ has an impaired capacity to favorably influence endothelial cell-monocyte adhesion or endothelial repair. It is unknown whether the loss in PON1 activity leads to alterations in other HDL constituents besides MDA that activate LOX-1. Whether HDL $L_{C A D}$ has decreased capacity to evoke cholesterol efflux from endothelial cells - possibly via MDA-related modification of apoA-I, resulting in attenuated SR-BI-dependent signaling - is also unknown. In addition, it is unknown whether endothelial cell signaling activated by cargo molecules such as S1P is altered in HDL $\mathrm{LAD}_{\text {. }}$ in endothelial cells; this causes cholesterol efflux that is sensed by SR-BI and initiates a signaling cascade involving the activation of Src family kinase(s), PI3K, and Akt, which phosphorylates eNOS at Ser1177 to increase enzyme activity (Figure 1A). These processes are dependent on the adaptor protein PDZK1, which binds to the extreme Cterminus of SR-BI. SR-BI-, Src-, and PI3Kdependent Erk MAPK activation is also required for eNOS activation by HDL. In addition, HDL-associated sphingosine-1- 
phosphate $(\mathrm{S} 1 \mathrm{P})$ and related molecules may activate the lysophospholipid receptor $\mathrm{S}_{1} \mathrm{P}_{3}$ to stimulate eNOS (16).

\section{Adverse endothelial effects of HDL from CAD patients}

Besler and colleagues now report that whereas HDL from healthy individuals (HDL $\left.\mathrm{Healthy}_{\text {H }}\right)$ causes an increase in bioavailable endothelium-derived NO, HDL from patients with stable CAD or acute coronary syndrome $\left(\mathrm{HDL}_{\mathrm{CAD}}\right)$ causes no increase or an actual decrease in NO (10). This is related to decreased activating eNOS Ser1177 phosphorylation and increased inactivating eNOS Thr495 phosphorylation by $\mathrm{HDL}_{\mathrm{CAD}}$ (Figure 1B). They also show that in an eNOS-dependent manner, $\mathrm{HDL}_{\text {Healthy }}$ promotes endothelial repair and blunts NF- $\kappa \mathrm{B}$ activation and VCAM-1 expression, thereby preventing endothelial cell-monocyte adhesion, whereas $\mathrm{HDL}_{\mathrm{CAD}}$ lacks these properties. They interrogated the basis for the adverse effects of $\mathrm{HDL}_{\mathrm{CAD}}$ on endothelial function, finding that total binding of $\mathrm{HDL}_{\mathrm{CAD}}$ to endothelium is decreased, but relative SR-BI-dependent binding is not altered. However, the researchers did not use endothelial cells to evaluate the effect of HDL $\mathrm{CAD}_{\mathrm{C}}$ on cholesterol efflux, which is critically involved in endothelial HDL SR-BI-mediated signaling to eNOS. When the efflux capacity of HDL is specifically altered, the relative activation of eNOS changes in parallel (17). If HDL $_{C A D}$ has a blunted capacity to promote cholesterol efflux from endothelial cells, this might help to explain the observed impairment in NO generation. This could be experimentally tested by the ex vivo addition of phosphatidylcholine (18) to enhance the endothelial cell cholesterol efflux capacity of HDL $\mathrm{CAD}_{\mathrm{C}}$ and determination of whether doing so restores the ability to generate NO.

Besler and colleagues additionally demonstrated that through a process involving the endothelial multiligand receptor known as lectin-type oxidized LDL receptor 1 (LOX-1), HDL $\mathrm{CAD}_{\mathrm{C}}$ activates endothelial PKC $\beta I I$, which in turn inhibits Akt-activating phosphorylation (Akt-Ser473) and eNOS-activating phosphorylation (eNOSSer1177) events and NO production (Figure $1 \mathrm{~B})$. Recognizing that endothelial LOX-1 is activated by oxidized lipids, they then evaluated the potential role of malondialdehyde (MDA) and found that MDA content is increased in $\mathrm{HDL}_{\mathrm{CAD}}$ compared with $\mathrm{HDL}_{\text {Healthy. }}$ They also show that the addition of MDA to HDL $\mathrm{Healthy}_{\text {blunts }}$ endothelial NO production and activates endothelial PKC $\beta$ II in a LOX-1-dependent fashion. Since HDL-associated paraoxonase 1 (PON1) diminishes MDA formation, they then evaluated PON1 and found that although its abundance is nearly doubled in $\mathrm{HDL}_{\mathrm{CAD}}$ versus $\mathrm{HDL}_{\text {Healthy, }}$ its enzyme activity is markedly decreased in $\mathrm{HDL}_{\mathrm{CAD}}$. The researchers further found that PON1 inactivation in $\mathrm{HDL}_{\text {Healthy }}$ leads to greater PKC $\beta$ II activation, decreased activating eNOS-Ser1177 phosphorylation and increased inactivating eNOS-Thr495 phosphorylation, blunted NO production, increased monocyte-endothelial cell adhesion, and impaired endothelial repair. These findings suggest a potential mechanistic link between decreased PON1 activity in HDL $\mathrm{CAD}_{\mathrm{D}}$ and exaggerated PKC $\beta$ II activation and impaired eNOS and endothelial function. Furthermore, they showed that HDL from Pon $1^{-/-}$mice fails to stimulate NO production or to antagonize endothelial inflammatory activation, and that supplementation of Pon $1^{-/-}$HDL with purified PON1 restores these functions (10). Since cholesterol-free lipoprotein particles consisting solely of apoA-I and phosphatidylcholine are sufficient to cause eNOS activation (17), similar to HDL $_{\mathrm{CAD}}$, the Pon $1^{-/}$HDL must contain component(s) that blunt eNOS activation such as MDA, which decreases efflux capacity via the modification of apoA-I (19). Although the available evidence implicates MDA, the modified component of $\mathrm{HDL}_{\mathrm{CAD}}$ and $\mathrm{Pon1}^{-/-} \mathrm{HDL}$ that is directly responsible for LOX-1- and PKC $\beta$ II-mediated eNOS inactivation requires further clarification. It is also unclear what causes the downregulation of PON1 activity in $\mathrm{HDL}_{\mathrm{CAD}}$, even though its abundance is increased. In any case, the findings by Besler et al. importantly indicate that HDL-associated PON1 has a major impact on endothelial function, which is consistent with the reported inverse relationship between PON1 activity and cardiovascular disease development (20).

\section{Remaining questions}

The work by Besler and colleagues has provided valuable evidence that HDL from CAD patients differs from HDL from healthy individuals in its capacity to invoke signaling in endothelial cells that induces eNOS activation and subsequent antiatherogenic and antiinflammatory processes. It also supports the concept that the car- diovascular impact of HDL is not simply related to its abundance. Furthermore, the findings suggest that assays of HDL action on endothelium may increase our ability to assign cardiovascular disease risk, and they may enhance our understanding of the outcomes of future trials testing HDLtargeted therapies. However, there are a number of remaining questions. Do the differences in endothelial intracellular signaling observed in cell culture in response to $\mathrm{HDL}_{\mathrm{Healthy}}$ versus $\mathrm{HDL}_{\mathrm{CAD}}$ reflect disparities in HDL-induced signaling in vivo? Are there differences in other critical endothelial cell phenotypes in vivo besides repair, such as leukocyte adhesion? The authors also appropriately point out that it remains unknown whether their findings represent a cause or a consequence of CAD. In this regard, the homogeneity of the functional defect in $\mathrm{HDL}_{\mathrm{CAD}}$ observed in this study is surprising, and additional cross-sectional studies with a wider spectrum of cardiovascular disease incidence and severity as well as prospective studies are now warranted. Combined with the recent study of atherosclerotic vascular disease and HDL macrophage cholesterol efflux (12), the work by Besler et al. indicates that we no longer need to ponder, but instead can conclude that measures of HDL function are clinically relevant. If we are earnest in our desire to harness the cardiovascular protective potential of HDL, we must go well beyond the quantification and even successful modification of HDL abundance, and reliably quantify and ultimately take therapeutic advantage of the bases for differences in HDL function.

\section{Acknowledgments}

This work was supported by NIH grant HL58888 (to P.W. Shaul). Additional support was provided by the Crystal Charity Ball Center for Pediatric Critical Care Research and the Lowe Foundation (to P.W. Shaul).

Address correspondence to: Philip W. Shaul, Department of Pediatrics, University of Texas Southwestern Medical Center, 5323 Harry Hines Blvd., Dallas, Texas 75390-9063, USA. Phone: 214.648.2015; Fax: 214.648.2096; E-mail: Philip.shaul@ utsouthwestern.edu.

\footnotetext{
1. Gordon DJ, Rifkind BM. High-density lipoprotein - the clinical implications of recent studies. NEngl JMed. 1989;321(19):1311-1316.

2. Hughes SD, Verstuyft J, Rubin EM. HDL deficiency in genetically engineered mice requires elevated LDL to accelerate atherogenesis. Arterioscler Thromb Vasc Biol. 1997;17(9):1725-1729.

3. Forrester JS, Shah PK. Emerging strategies for
} 
increasing high-density lipoprotein. Am J Cardiol. 2006;98(11):1542-1549.

4. Williams KJ, Feig JE, Fisher EA. Rapid regression of atherosclerosis: insights from the clinical and experimental literature. Nat Clin Pract Cardiovasc Med. 2008;5(2):91-102.

5. Mendez AJ. The promise of apolipoprotein A-I mimetics. Curr Opin Endocrinol Diabetes Obes. 2010;17(2):171-176.

6. Khera AV, Rader DJ. Discovery and validation of new molecular targets in treating dyslipidemia: the role of human genetics. Trends Cardiovasc Med. 2009;19(6):195-201.

7. Briel M, et al. Association between change in high density lipoprotein cholesterol and cardiovascular disease morbidity and mortality: systematic review and meta-regression analysis. BMJ. 2009;338:b92.

8. Singh IM, Shishehbor MH, Ansell BJ. High-density lipoprotein as a therapeutic target: a systematic review. JAMA. 2007;298(7):786-798.

9. Barter PJ, et al. Effects of torcetrapib in patients at high risk for coronary events. $N$ Engl J Med. 2007;357(21):2109-2122.

10. Besler C, et al. Mechanisms underlying adverse effects of HDL on eNOS-activating pathways in patients with coronary artery disease. J Clin Invest. 2011;121(7):2693-2708.

11. Duffy D, Rader DJ. Update on strategies to increase HDL quantity and function. Nat Rev Cardiol. 2009;6(7):455-463.

12. Khera AV, et al. Cholesterol efflux capacity, highdensity lipoprotein function, and atherosclerosis. N Engl J Med. 2011;364(2):127-135

13. Mineo C, Deguchi H, Griffin JH, Shaul PW. Endothelial and antithrombotic actions of HDL. Circ Res. 2006;98(11):1352-1364.

14. Yuhanna IS, et al. High-density lipoprotein binding to scavenger receptor-BI activates endothelial nitric oxide synthase. Nat Med. 2001;7(7):853-857.

15 . Seetharam D, et al. High-density lipoprotein promotes endothelial cell migration and reendothelialization via scavenger receptor-B type I. Circ Res.
2006;98(1):63-72

16. Saddar S, Mineo C, Shaul PW. Signaling by the highaffinity HDL receptor scavenger receptor B type I. Arterioscler Thromb Vasc Biol. 2010;30(2):144-150.

17. Assanasen C, et al. Cholesterol binding, efflux, and a PDZ-interacting domain of scavenger receptorBI mediate HDL-initiated signaling. J Clin Invest. 2005;115(4):969-977.

18. Yancey PG, et al. High density lipoprotein phospholipid composition is a major determinant of the bidirectional flux and net movement of cellular free cholesterol mediated by scavenger receptor BI. J Biol Chem. 2000;275(47):36596-36604

19. Shao B, et al. Modifying apolipoprotein A-I by malondialdehyde, but not by an array of other reactive carbonyls, blocks cholesterol efflux by the ABCA1 pathway. J Biol Chem. 2010;285(24):18473-18484.

20. Regieli JJ, et al. Paraoxonase variants relate to 10 -year risk in coronary artery disease: impact of a high-density lipoprotein-bound antioxidant in secondary prevention. J Am Coll Cardiol. 2009;54(14):1238-1245.

\title{
Adenosine: front and center in linking nutrition and metabolism to neuronal activity
}

\author{
Robert W. Greene
}

Department of Psychiatry, University of Texas Southwestern Medical Center and Dallas VA Medical Center, Dallas, Texas, USA.

\begin{abstract}
Many individuals with epilepsy benefit from consuming a ketogenic diet, which is similar to the more commonly known Atkins diet. The underlying molecular reason for this has not been determined. However, in this issue of the JCI, Masino et al. have elucidated the mechanism responsible for the antiepileptic effects of the ketogenic diet in mice. The diet is shown to decrease expression of the enzyme adenosine kinase (Adk), which is responsible for clearing the endogenous antiepileptic agent adenosine (Ado) from the extracellular CNS space. Decreased expression of Adk results in increased extracellular Ado, activation of inhibitory Ado $A_{1}$ receptors, and decreased seizure generation, the desired therapeutic effect. The authors' work serves to emphasize the importance of controlling Adk expression, not only as the mechanism of action of the ketogenic diet, but also as a potential target of future therapies.
\end{abstract}

Epilepsy is a brain disorder characterized by recurrent seizures that last a few seconds to up to 15 minutes, but seldom longer. The seizures are a result of synchronized electrical discharge by a large proportion of CNS neurons. They usually originate from one or more hyperexcitable neuronal foci and propagate to both surrounding neuronal tissue and downstream targets of the excited neurons. Most therapeutic approaches involve pharmacological agents targeted toward reducing the excitability of the irritable seizure focus and/or the propagation of the synchronized discharges. However,

Conflict of interest: The author has declared that no conflict of interest exists.

Citation for this article: J Clin Invest. doi:10.1172/ JCI58391. these agents often cause undesirable side effects, and in some individuals with epilepsy, the seizures are refractory to pharmacological agents. An alternative therapy that has shown some success in this regard is the ketogenic diet.

The ketogenic diet, similar to the more commonly known Atkins diet, provides a metabolic treatment for epilepsy. The highfat, low-carbohydrate diet forces ketonebased rather than glucose-based metabolism and has well-demonstrated antiepileptic efficacy (1). However, the mechanism responsible for the anticonvulsant effect of a ketogenic diet remains to be characterized. In this issue of the JCI, Masino et al. convincingly demonstrate that a ketogenic diet works by modifying the availability of one of the best-characterized and effica- cious endogenous antiepileptic compounds in mammals, adenosine (Ado) (2). The authors show that in mice, the ketogenic diet is associated with decreased expression of the enzyme responsible for removal of Ado from the CSF, adenosine kinase (Adk). As a result, there is an increase in Ado in the CSF that in turn activates Ado $A_{1}$ receptors $\left(\right.$ Ado $\left._{1} R s\right)$. This activation was found to be both necessary and sufficient for the antiepileptic effect of the ketogenic diet. The work of Masino et al. (2) serves to emphasize the therapeutic potential of controlling Adk expression in epilepsy and the need for better understanding of this control.

\section{Ado: the endogenous antiepileptic agent}

An endogenous antiepileptic agent should have, at the least, the following two properties. First, its levels should be increased in brain tissue by conditions that predispose to seizure generation and/or by seizure activity itself. Second, when released, it should act to reduce the likelihood of the seizure activity.

Conditions that promote seizures include hypoxia of nervous tissue; hypoglycemia; increased levels of potassium or other excitogenic substances, such as glutamate (for example, as might be released as a result of acute brain trauma), in the brain extracellular medium; or simply an 